\title{
An Analysis of Anatomic Variations of the Sphenoid Sinus and Its Relationship to the Internal Carotid Artery
}

\author{
Myrian Marajó Dal Secchi ${ }^{1}$ Ricardo Landini Lutaif Dolci ${ }^{1} \quad$ Reginaldo Teixeira $^{2}$ Paulo Roberto Lazarini ${ }^{1}$ \\ ${ }^{1}$ Department of Otorhinolaryngology, Faculdade de Ciências Médicas \\ da Santa Casa de São Paulo, São Paulo, SP, Brazil \\ 2 Department of Radiology, Irmandade da Santa Casa da Misericórdia \\ de Santos, Santos, SP, Brazil \\ Address for correspondence Myrian Marajó Dal Secchi, PhD, \\ Department of Otorhinolaryngology, Faculdade de Ciências Médicas, \\ Santa Casa de São Paulo, Av. Ana Costa 254, CJ 72, Santos, \\ SP 11060-000, Brazil (e-mail: dalsecchi@uol.com.br).
}

Int Arch Otorhinolaryngol 2018;22:161-166.

\begin{abstract}
Introduction The sphenoid sinus (SS) has a high variability; its anatomical relations and variations must be well understood prior to the expanded endoscopic surgery (EES) at the skull base via the endonasal transsphenoidal approach. A feared complication is injury to the internal carotid artery (ICA).

Objective To evaluate the anatomic variations of the SS and its relationship to the ICA using computed tomography (CT).

Methods Cross-sectional retrospective study. Analysis of 90 patients' CT scans on axial, coronal and sagittal planes with $1 \mathrm{~mm}$ slices, evaluating lateral and posterior extensions of pneumatization of the SS, deviation of the sphenoid septum, presence of septations and their relationship to the parasellar and paraclival segments of the

\section{Keywords}

- carotid artery

- internal

- paranasal sinuses/ surgery

- skull base

- sphenoid sinus

- sphenoid bone

- tomography

- X-ray computed

internal carotid artery (psICA and pcICA, respectively).

Results The association between the protrusions of the psICA and the pICA was statistically significant $(p<0.001)$, as was the association between the lateral extension of pneumatization of the SS and the protrusion of the psICA $(p=0.014)$. The presence of the posterior extension of pneumatization of the SS and protrusion of the pcICA occurred in $46 \%$ of the cases. Deviation of the sphenoid septum in the direction of the pcICA was present in $14 \%$ and dehiscence of the pclCA was seen in $3.6 \%$ of the cases.

Conclusion Using the CT scan to recognize the type of extensions of pneumatization of the SS, the deviation of the sphenoid septum, and the presence of septations is beneficial to identify accurately the ICA and to reduce the risk of injury to it.
\end{abstract}

\section{Introduction}

Surgical access to the anterior region of the skull base via transsphenoidal surgery is commonly used by otorhinolaryngologists. Radiologic assessment of this region pre- and intraoperatively is fundamental for surgeons to identify the neurovascular structures and their anatomic relationships, thereby reducing possible complications during and after surgical intervention. Computed tomography (CT) of the

received

April 3, 2017

accepted

September 3, 2017

published online

October 25, 2017 paranasal sinus is the best method for studying the anatomy and variation of this region. ${ }^{1-3}$

The sphenoid sinus (SS) may show varying degrees and directions of pneumatization. Its various extensions and critical relationship to neurovascular structures make intimate knowledge of the anatomy of the region and its variations something essential. The internal carotid artery (ICA) is the anatomic structure that must be assessed in the region of the skull
Copyright @ 2018 by Thieme Revinter Publicações Ltda, Rio de Janeiro, Brazil

\section{License terms}

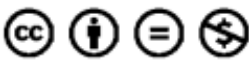

10.1055/s-0037-1607336. ISSN $1809-9777$. 
base before and during the surgery. Axial and coronal CT scans are indispensable for disclosing the location of the ICA. ${ }^{4-6}$

In the clinical practice, $\mathrm{CT}$ assessment prior to surgery allows the relationships between the SS and the ICA, and between the lesion and the ICA, to be established. This is fundamental, for example, for planning the endoscopic surgical approach and for safe removal of the lesion with ICA control., ${ }^{6,7}$

The objective of the present study is to use the CT to analyze the lateral and posterior extensions of pneumatization of the SS and their relationship with parasellar and paraclival segments of the internal carotid artery (psICA and pcICA, respectively). The study also aims to determine the frequency of possible local anatomic variations of the SS in relation to these segments of the ICA adjacent to it.

\section{Methods}

This study was approved by the Research Ethics Committee of the Institution (CAAE 14725713.7.0000.5479). A crosssectional retrospective study of 90 paranasal sinus CT scans was performed in patients clinically indicated for this supplementary exam. Inclusion criteria: tomography scans of individuals older than 18 years of age, with rhinosinusal symptoms and request from physician ordering tomography assessment of the nose and paranasal sinus. Exclusion criteria: tomography scans identifying individuals with facial bone fractures, rhinosinusal neoplasms, or rhinosinusitis of the posterior paranasal sinus.

Tomography scans were performed using an electro spin resonance (ESR) Hispeed helicoidal tomography manufactured by GE, model CT/e (Computed Tomography/helicoidal), series 1676 HMS (GE, Boston, MA, USA). The analysis of the variables was performed using the eFilm 2.1 computer software program (Merge Healthcare Inc., Chicago, IL, USA).

The exam protocol included the acquisition of images using the helicoidal technique on axial, coronal and sagittal planes, with reconstruction of $1.0 / 1.0 \mathrm{~mm}$ to $1.0 / 1.0 \mathrm{~mm}$ slices (slice thickness/increment). The first image on coronal or axial plane, which identifies the structures of the SS and the ICA, was used for the tomographic analysis in the anterior to posterior and superior to inferior directions, respectively.

Based on the images acquired by the tomography scans, the following variables were assessed: the type of pneumatization of the SS, the extensions of the sellar type pneumatization, the anatomic variations of the ICA, the presence of septations, and the position of the sphenoidal septum.

\section{Classification of Anatomic Variations of the SS}

- Classification of the type of pneumatization of the SS in relation to the sella turcica into: conchal, presellar and sellar. Analysis of the sagittal plane.

- Classification of the extensions of sellar-type pneumatization into: lateral (lesser wing, greater wing, pterygoid and complete (greater wing and pterygoid process) (- Fig. 1A, -Fig. 1B) and posterior (critical and non-critical) (-Fig. 1C, -Fig. 1D). Analysis of the lateral extensions on coronal plane and the posterior extensions on axial plane. Two types of posterior extension were considered according to the width between the posterior boundary of the SS and the clivus identified on the axial slices. The type was defined as critical for thickness $<2 \mathrm{~mm}$ and noncritical for clivus thickness $\geq 2 \mathrm{~mm}$.

\section{Classification of Anatomic Variations of the Parasellar and Paraclival Segment of the ICA in Relation to the Lateral and Posterior Extensions of the SS}

- Absence of protrusion, with protrusion, and presence of dehiscence. Analysis of the psICA on coronal plane (-Fig. 2A) and the pcICA on axial plane (-Fig. 2B). Protrusion was defined as $>50 \%$ projection of the structure into the sphenoid sinus and dehiscence as the absence of visible bone separating the ICA from the SS along its path.

\section{Classification Regarding the Presence or Absence of Septations}

Analysis on Axial and Coronal Planes (-Fig. 3A, -Fig. 3B)

- Classification of the sphenoidal septum: on the sagittal plane, with deviation to the right or left and in the direction of the ICA. Analysis of axial plane (-Fig. 3C, - Fig. 3D).

The chi-square test was used for statistical analysis of the data, and a $5 \%$ level of significance was adopted.

\section{Results}

Total 90 tomographic imaging exams were studied in patients whose ages ranged from 19 to 84 years with a mean of 46 years $(S D=16.4)$ and median of 44 years. The sample comprised $33 \%$ males and $67 \%$ females.

The analysis of the pneumatization of the SS in relation to the sella turcica revealed a predominance of the sellar type in $98 \%$ of the patients, the presellar type in $2 \%$ of patients and no cases of the conchal type. Lateral extension of pneumatization of the SS for the lesser wing of the sphenoid was found in $13 \%$ of the individuals, with the greater wing type in $47 \%$, the pterygoid type in only $1 \%$, the complete type in $23 \%$ and absent in $16 \%$. The presence of posterior extension was found in $78 \%$ of the individuals, comprising critical type pneumatization in $42 \%$, non-critical type in $36 \%$, and absent in $22 \%$.

Protrusion of the psICA was found in $26 \%$ of the individuals and it was absent in $74 \%$, whereas protrusion of the pcICA was noted in $35 \%$ and absent in $64 \%$ of the patients. Dehiscence of the pcICA was observed in four cases (3.6\%), two of which were right side and two were left sides.

The statistical analysis using the chi-square test for the association between protrusions of the pcICA and psICA was statistically significant $(p<0.001)$, as was the association between the lateral extension of pneumatization of the SS and protrusion of the psICA $(p=0.014)$ ( $~-$ Table 1$)$. A presence of protrusion of the pcICA in individuals of the posterior extension of pneumatization of the SS was present in $46 \%$ and absent in $54 \%$ of the cases.

Septations were present in $39 \%$ and absent in $61 \%$ of the individuals. On the chi-square test, no significant difference 

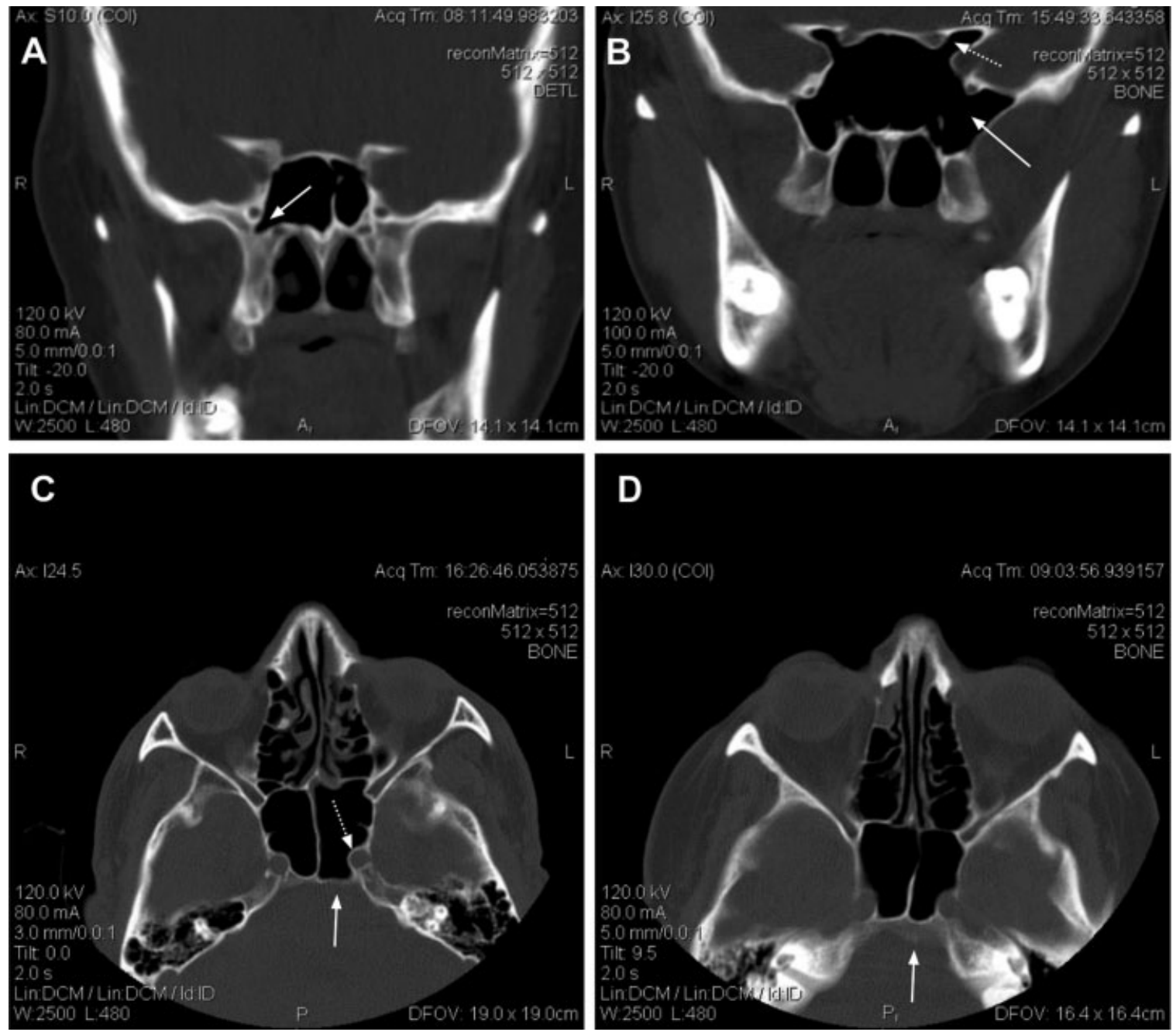

Fig. 1 Coronal plane: (A) lateral extension to greater wing (arrow), (B) lateral extension to greater wing and pterygoid process (complete) (arrow) and lesser wing (dotted line). Axial plane: (C) critical posterior extension (arrow) and protrusion of paraclival segment (dotted line), (D) non-critical posterior extension (arrow).
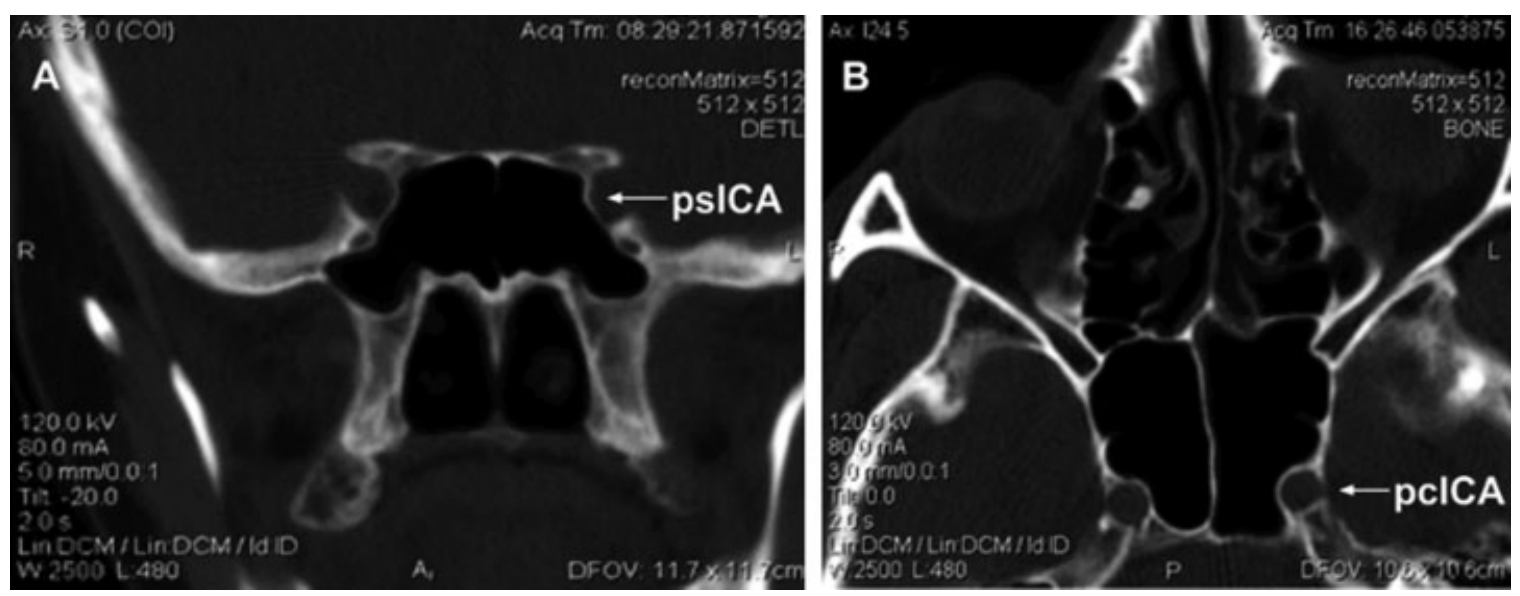

Fig. 2 (A) Coronal plane, protrusion of parasellar segment of the internal carotid artery (psICA) and lateral extension of the sphenoid sinus. (B) Axial plane, protrusion of paraclival segment of the internal carotid artery (pcICA) and posterior extension of the sphenoid sinus (SS). 

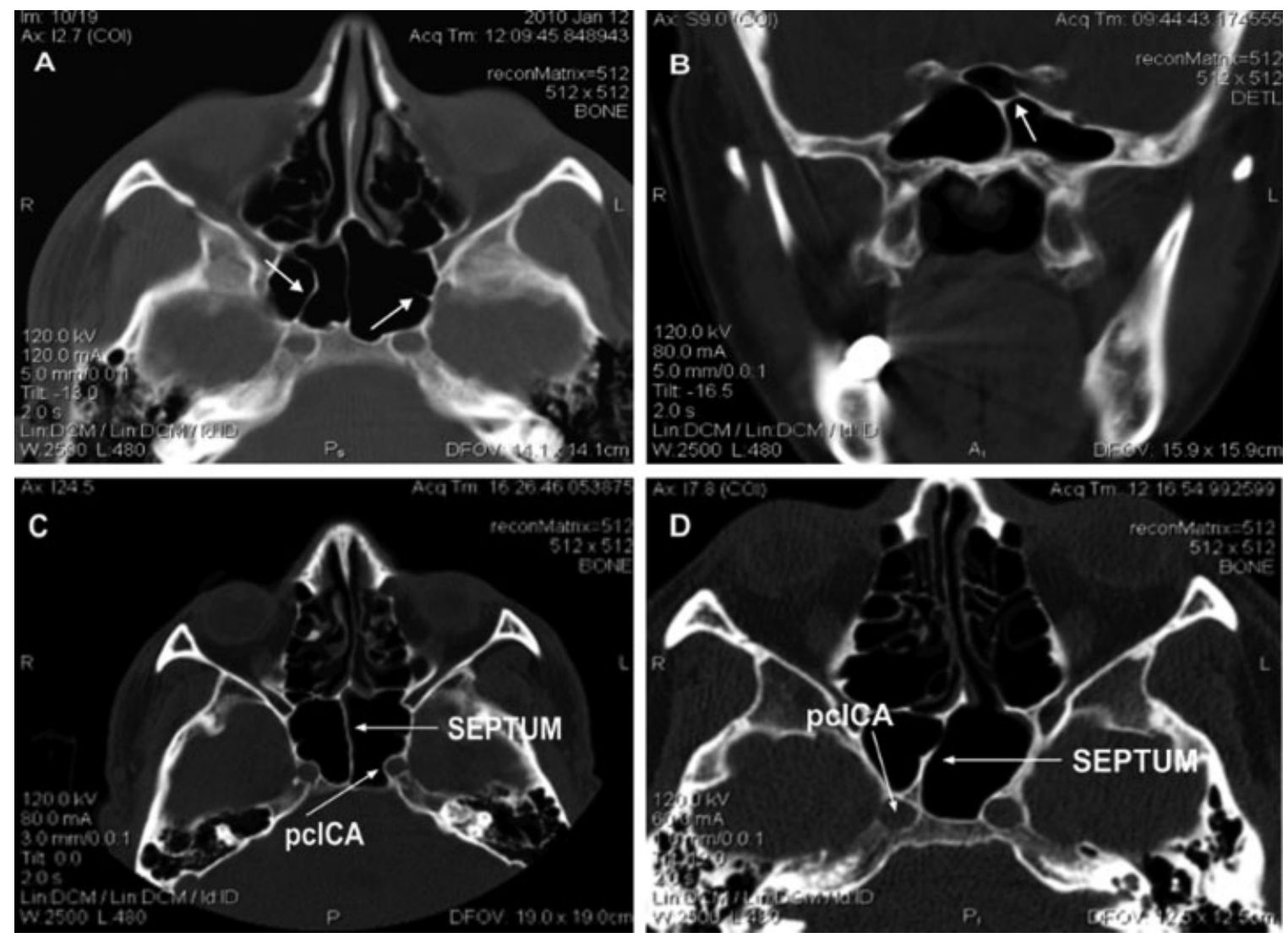

Fig. 3 (A) Axial plane, presence of septations (arrows), (B) Coronal plane, presence of septation to the left (arrow), (C) Axial plane, sphenoid septum on sagittal plane (arrow) and protrusion of paraclival segment (pcICA) and (D) Axial plane, deviation of the sphenoid septum in the direction of the paraclival segment of the internal carotid artery (pclCA).

was observed between individuals exhibiting septations with or without posterior extension $(p=0.908)$.

The deviation of the sphenoid septum either to the right or left was found in $60 \%$ of the individuals; on the sagittal plane, in 26\%; deviation in the direction of the ICA, in $14 \%$, and in one or more septum, in 5\% of the CT scans. In those individuals exhibiting posterior extension, the position of the sphenoid septum most commonly observed was deviation. No significance was reached on the chi-square test $(p=0.289)$ (-Table 2).

\section{Discussion}

Extensive pneumatization of the SS can be associated with irregularities in the sinus wall featuring protrusions and

Table 1 Percentage distribution of lateral extension of pneumatization of sphenoid sinus in relation to protrusion of the psICA

\begin{tabular}{|l|l|l|l|l|l|}
\hline \multicolumn{2}{|l|}{ Extension } & \multicolumn{2}{|l|}{$\begin{array}{l}\text { Protrusion of } \\
\text { psICA }\end{array}$} & Total & $p$ \\
\cline { 3 - 4 } \multicolumn{2}{|l|}{} & absent & present & & \\
\hline \multirow{2}{*}{ Lateral } & absent & 46 & 54 & 100 & 0.014 \\
\cline { 2 - 5 } & present & 17 & 83 & 100 & \\
\hline
\end{tabular}

Abbreviation: psICA, parasellar segment of internal carotid artery. recesses, such as prominences of the ICA. Computed tomography can help to identify the relationship of the ICA with the lateral and posterior extensions of pneumatization of the SS, and alert the surgeons to regions for possible injury occurrences. ${ }^{8,9}$

The present study evaluated the degree and types of pneumatization of the SS and their relationship with the paraclival (pcICA) and parasellar (psICA) segments of the ICA. These segments of the ICA are adjacent to the SS and can be damaged during expanded endoscopic approaches (EEA) to the skull base. $5,7,10,11$

In the literature, protrusion of the ICA ranges from 8 to $70 \%{ }^{12}$ In the present study, however, the presence of psICA protrusion occurred in $26 \%$ of the individuals, while the presence of protrusion of the pcICA was detected in 35\%. In this study, a statistically significant association was found between protrusion of the psICA and pcICA $(p<0.001)$. Dehiscence in the pcICA was found in $3.6 \%$ of the individuals compared with $5 \%$ of cases identified by Meloni et al, ${ }^{1} 1.5 \%$ by Kazkayasi et al, ${ }^{3} 14.4 \%$ by Johnson et al, ${ }^{4}$ and $5.3 \%$ of cases by Unal et al. ${ }^{13}$ In the current study, the pcICA and psICA were evaluated separately and no cases of dehiscence in the psICA was detected. Axial and coronal CT scans are indispensable for disclosing the location of these segments adjacent to the SS. ${ }^{4-6}$

Assessment of pneumatization of the SS helps surgeons to elect the optimum approach and determine the impact of anatomic variations on the surgery. ${ }^{9,14,15}$ In this study, the 
Table 2 Percentage distribution of the position of sphenoid septum, confirmed by paranasal sinus CT, according to the presence or absence of posterior extension of pneumatization of sphenoid sinus

\begin{tabular}{|l|l|l|l|l|l|l|}
\hline \multicolumn{2}{|l|}{ Extension } & $\begin{array}{l}\text { Sagittal } \\
\text { plane }\end{array}$ & Deviation & $\begin{array}{l}\text { ICA path } \\
\text { deviation }\end{array}$ & Total \\
\hline \multirow{3}{*}{ Posterior } & present & 23 & 60 & 17 & 78 & 0.289 \\
\cline { 2 - 7 } & absent & 35 & 60 & 5 & 14 & 22 \\
\hline Total & & 26 & 60 & 100 & \\
\hline
\end{tabular}

Abbreviations: CT, computed tomography; ICA, internal carotid artery.

sellar type was the most frequent, found in $98 \%$ of the cases, the presellar type occurred in $2 \%$ of cases, while no instances of the conchal type were detected, mirroring the results of the study by Wang et al. ${ }^{8}$ On CT, the configuration of the SS can be identified on sagittal plane, where the sellar type facilitates EEAs, with lower risk of injury to the neurovascular structures surrounding the SS, although there is less bone to remove during exposure of the operative region. However, it becomes easier to identify the landmarks, whereas for the presellar type, the SS walls tend to be thicker and more difficult for transsphenoidal approaches. Lateral and posterior extension may occur in sellar-type pneumatization, and a classification of these extensions is important as a guide to the approaches extending beyond the sella turcica.

In the present study, the greater wing lateral type extension was the most frequently found, in contrast to Wang et $\mathrm{al}^{8}{ }^{8}$ who predominantly found the complete type when the extension is to the greater wing and the pterygoid process. Both types facilitate access to the cavernous sinus, middle cranial fossa and petrous apex because there is less bone to remove. These disparities may be explained by individual variations in the population. The posterior extension, in turn, was identified in $78 \%$ of all CTs, comprising $42 \%$ critical and $36 \%$ non-critical types, demonstrating the high incidence of this anatomic variation. Haetinger et $\mathrm{al}^{16}$ detected posterior extension in $69 \%$ of the cases and $44 \%$ of these were of the critical type. The critical type facilitates access to lesions located in the clivus and petrous apex, but there is an associated risk of perforation of the clivus due to the bone thickness of this region. ${ }^{16}$ This classification of the lateral and the posterior extensions in the $\mathrm{CT}$ provides a guide to evaluate the degrees and directions of the pneumatization of the SS and its relationship with the psICA and the pcICA.

In this study, extensions of pneumatization into the lesser wing were considered lateral type extensions because this definition better fits the classification of the lateral extensions on CT scans, given the location of this extension in the superior and lateral region of the SS and its relationship with both the optic nerve and psICA. On the other hand, Wang et $\mathrm{al}^{7}$ classified the lesser wing type separately from the lateral extensions. This extension was found in $13 \%$ of the current cases.

A statistically significant association was found between pneumatization of the lateral extension and the presence of protrusion of the psICA ( $p=0.014)$. Pneumatization of the posterior extension and presence of protrusion of the psICA were relevant in $46 \%$ of the cases. This association disclosed on CT alerts surgeons of the impact of these variations on the surgery, whose outcome is linked to the increase of the SS to beyond normal anatomic limits. Thus, preoperative CT can identify areas with potential risk of complication, such as ICA injury in the SS featuring these extensions.

The local variations of the SS identified on CT include deviations of the sphenoid septum and the presence of septations in the SS. ${ }^{2}$ The computed tomography provides preoperative anatomic information regarding the course of the ICA to these local variations of the SS. Injury to the ICA can thus be avoided during surgery. In this study, for those individuals exhibiting posterior extension, the position of the septum most commonly observed was deviation, found on $60 \%$ of scans, although this association was not statistically significant ( $p=0.289$ ), 14\% of the individuals had deviation of the septum in the direction of the pcICA, and septations were identified in $39 \%$ of the cases. No statistically significant difference between individuals exhibiting septations with and without posterior extensions was found ( $p=0.908$ ).

The sphenoidal septum is often deflected to one side or the other, and can attach to the bone wall that protects the ICA, where fracture of the septum in order to gain access to the ICA can damage this artery. ${ }^{2,3,9,13,17}$ There is a potential risk of injury when septations are located near the prominence of the ICA. Therefore, identifying the artery is crucial to keep a safe distance during tumor resection., $9,18-20$

An analysis of the anatomic variations of the SS observed on CT scans and their relationships with the segments of the ICA adjacent to the SS allows for more specific descriptions by the radiologists regarding patients who require EEA to the skull base, where key anatomic features are highlighted such as: the types of pneumatization of the SS and their extensions; irregularities in the walls of the psICA and pcICA; position of the septum in relation to the ICA and presence of septations in the SS. This information acquired, in conjunction with a preoperative assessment of imaging exams by the surgeon and their team, alert to all the aspects outlined above. Therefore, identifying the anatomic boundaries of this region for surgery can be done with more accuracy and reliability.

\section{Conclusion}

The evaluation of the SS preoperatively using a CT scan can aid surgeons to be prepared before performing an endoscopic skull base surgery. Particularly, it provides essential tools to know what will be found at the surgery, avoiding any 
iatrogenic lesion, such as damage to the ICA or noble structures, and thus allowing to better plan the surgery. The anatomic variations and their relationship should be reported by the radiologist.

Preoperative recognition of the type of extensions of pneumatization of the SS, deviation of the sphenoid septum and presence of septations are beneficial to identify safely the psICA and pcICA adjacent to the SS. This information may be helpful if intraoperative surgical navigation is used.

\section{Conflict of Interest}

No conflicts of interest have been declared by the authors.

\section{Informed Consent}

This study was retrospective; thus, the informed consent was not required.

\section{References}

1 Meloni F, Mini R, Rovasio S, Stomeo F, Teatini GP. Anatomic variations of surgical importance in ethmoid labyrinth and sphenoid sinus. A study of radiological anatomy. Surg Radiol Anat 1992;14(01):65-70

2 Sirikci A, Bayazit YA, Bayram M, Mumbuç S, Güngör K, Kanlikama M. Variations of sphenoid and related structures. Eur Radiol 2000; 10(05):844-848

3 Kazkayasi M, Karadeniz Y, Arikan OK. Anatomic variations of the sphenoid sinus on computed tomography. Rhinology 2005;43 (02):109-114

4 Johnson DM, Hopkins RJ, Hanafee WN, Fisk JD. The unprotected parasphenoidal carotid artery studied by high-resolution computed tomography. Radiology 1985;155(01):137-141

5 Kennedy DW, Zinreich SJ, Hassab MH. The internal carotid artery as it relates to endonasal sphenoethmoidectomy. Am J Rhinol 1990;4(01):7-12

6 Labib MA, Prevedello DM, Carrau R, et al. A road map to the internal carotid artery in expanded endoscopic endonasal approaches to the ventral cranial base. Neurosurgery 2014;10 (Suppl 3):448-471, discussion 471

7 Gardner PA, Tormenti MJ, Pant H, Fernandez-Miranda JC, Snyderman $\mathrm{CH}$, Horowitz MB. Carotid artery injury during endoscopic endonasal skull base surgery: incidence and outcomes. Neurosurgery 2013;73(2, Suppl Operative)ons261-ons269, discussion ons269-ons270

8 Wang J, Bidari S, Inoue K, Yang H, Rhoton A Jr. Extensions of the sphenoid sinus: a new classification. Neurosurgery 2010;66(04): 797-816

9 Hamid O, El Fiky L, Hassan O, Kotb A, El Fiky S. Anatomic variations of the sphenoid sinus and their impact on trans-sphenoid pituitary surgery. Skull Base 2008;18(01):9-15

10 Amin SM, Nasr AY, Saleh HA, Foad MM, Herzallah IR. Endoscopic orientation of the parasellar region in sphenoid sinus with illdefined bony landmarks: an anatomic study. Skull Base 2010;20 (06):421-428

11 Anusha B, Baharudin A, Philip R, Harvinder S, Shaffie BM. Anatomical variations of the sphenoid sinus and its adjacent structures: a review of existing literature. Surg Radiol Anat 2014;36(05): 419-427

12 Kantarci M, Karasen RM, Alper F, Onbas O, Okur A, Karaman A. Remarkable anatomic variations in paranasal sinus region and their clinical importance. Eur J Radiol 2004;50(03):296-302

13 Unal B, Bademci G, Bilgili YK, Batay F, Avci E. Risky anatomic variations of sphenoid sinus for surgery. Surg Radiol Anat 2006;28 (02):195-201

14 Vaezi A, Cardenas E, Pinheiro-Neto C, et al. Classification of sphenoid sinus pneumatization: relevance for endoscopic skull base surgery. Laryngoscope 2015;125(03):577-581

15 Hammer G, Radberg C. The sphenoidal sinus. An anatomical and roentgenologic study with reference to transsphenoid hypophysectomy. Acta Radiol 1961;56:401-422

16 Haetinger RG, Navarro JA, Liberti EA. Basilar expansion of the human sphenoidal sinus: an integrated anatomical and computerized tomography study. Eur Radiol 2006;16(09):2092-2099

17 Aksoy F, Yenigun A, Goktas SS, Ozturan O. Association of accessory sphenoid septa with variations in neighbouring structures. J Laryngol Otol 2017;131(01):51-55

18 Fernandez-Miranda JC, Prevedello DM, Madhok R, et al. Sphenoid septations and their relationship with internal carotid arteries: anatomical and radiological study. Laryngoscope 2009;119(10): 1893-1896

19 Poirier J, Duggal N, Lee D, Rotenberg B. Sphenoid sinus septations: unpredictable anatomic landmarks in endoscopic pituitary surgery. J Otolaryngol Head Neck Surg 2011;40(06):489-492

20 Dasar U, Gokce E. Evaluation of variations in sinonasal region with computed tomography. World J Radiol 2016;8(01):98-108 